\title{
New Physics in ultraperipheral collisions at the LHC
}

\author{
S. I. Godunov ${ }^{1,2, *}$, V. A. Novikov ${ }^{1,3,4, * *}, A . N$ Rozanov $^{1,5, * * *}, M . I$. Vysotsky $^{1,3,4, * * * *}$, and E. V. \\ Zhemchugov ${ }^{1,4, \dagger}$ \\ ${ }^{1}$ Institute for Theoretical and Experimental Physics, 117218, Moscow, Russia \\ ${ }^{2}$ Novosibirsk State University, 630090, Novosibirsk, Russia \\ ${ }^{3}$ National Research University Higher School of Economics, 101978, Moscow, Russia \\ ${ }^{4}$ Moscow Engineering Physics Institute, 115409, Moscow, Russia \\ ${ }^{5}$ The Center for Particle Physics of Marseilles, CPPM-IN2P3-CNRS-AMU, F-13288, Marseille, France
}

\begin{abstract}
We present a model-independent approach for New Physics searches in ultraperipheral collisions at the LHC. The case of long-lived charged particles is considered and it is shown that it is possible to find them even with the already available LHC data.
\end{abstract}

\section{Introduction}

Common situation in searching for SUSY particles can be described in the following way. No events found in the expected signal region so the limits are set on the fiducial cross section. These limits are then reinterpreted in the framework of the particular SUSY model to get bounds on particle masses and other model parameters. To do that one has to know the production cross section to compare it with the experimental limit. That implies the scan over model parameters space. Therefore most of the results are model dependent and sensitive to additional New Physics (extra Higgses, $Z^{\prime}$, etc). Ultraperipheral collisions (UPC) ${ }^{1}$ provide us with a model-independent method of searching for new particles in photon fusion since photon coupling is universal being defined only by particle charge.

We suggest to look for long-lived charged particles, for instance, quasistable charginos $\tilde{\chi}_{1}$ that exist in some SUSY scenarios (see [1, §110.7]). In what follows we consider only charginos that pass through the muon system. There are many searches for long-lived particles (LLP) in inelastic processes at the LHC [2-13]. However, they are strongly depend on the model due to the reasons described above.

In UPC, production cross section is much smaller than in inelastic processes, though the process is much cleaner. In addition to that it is possible to detect in forward detectors (ATLAS Forward Proton Detector (AFP) [14] or CMS-TOTEM Precision Proton Spectrometer [15]) protons from the event since they remain intact after interaction. Therefore, full kinematics of the event can be reconstructed allowing for additional background suppression.

\footnotetext{
*e-mail: sgodunov@itep.ru

**e-mail: novikov@itep.ru

***e-mail: rozanov@cppm.in2p3.fr

****e-mail: vysotsky@itep.ru

†e-mail: zhemchugov@itep.ru

${ }^{1}$ These are events where colliding particles remain intact after the collision and production of new particles occurs in fusion of photons emitted by these particles.
} 
It is worth noting that the production cross section in ion-ion collisions is enhanced as $\sim Z^{4}$ in comparison to the proton-proton case. For $Z=82$ we have the factor $4.5 \cdot 10^{7}$. However, the luminosity collected in lead-lead collisions is $6.6 \cdot 10^{7}$ times less than the $p p$ one. Therefore, one can expect that both ion-ion and $p p$ data are equally promising for searches for New Physics appearing in photon-photon fusion.

The present talk is based on the paper [16].

\section{Production cross section in the framework of EPA}

Due to large LHC energy we can treat the electromagnetic field of the source particle as real photons distributed according to a well known spectrum. This approximation is known as Equivalent Photon Approximation (EPA) and for the LHC it works really well [17]. It greatly simplifies the derivation of analytical results. In this section we provide formulas for the total and fiducial (with the account of kinematic cuts) cross sections.

The EPA provides the momentum distribution of photons:

$$
n\left(\vec{q}_{\perp}, \omega\right) \mathrm{d}^{2} q_{\perp} \mathrm{d} \omega=\frac{Z^{2} \alpha}{\pi^{2}} \frac{\vec{q}_{\perp}^{2}}{\omega\left(\vec{q}_{\perp}^{2}+\frac{\omega^{2}}{\gamma^{2}}\right)^{2}}\left|F\left(\vec{q}_{\perp}^{2}+\frac{\omega^{2}}{\gamma^{2}}\right)\right|^{2} \mathrm{~d}^{2} q_{\perp} \mathrm{d} \omega
$$

where $\omega$ is the photon energy in the laboratory frame, $\vec{q}_{\perp}$ is the transverse component of the photon momentum, $\gamma$ is the Lorentz factor of the source particle, $F$ is the form factor originating from the vertex involving the particle which emits photons. For protons the Dirac form factor is a very good approximation [18] while for ions there are experimentally measured form factors [19].

For the total chargino production cross section in EPA we get:

$$
\sigma\left(N N \rightarrow N N \tilde{\chi}_{1}^{+} \tilde{\chi}_{1}^{-}\right)=\int_{0}^{\infty} \int_{0}^{\infty} \sigma\left(\gamma \gamma \rightarrow \tilde{\chi}_{1}^{+} \tilde{\chi}_{1}^{-}\right) n_{N}\left(\omega_{1}\right) n_{N}\left(\omega_{2}\right) \mathrm{d} \omega_{1} \mathrm{~d} \omega_{2}
$$

where $N$ is the colliding particle, $n_{N}(\omega)$ is its equivalent photon spectrum ${ }^{2}$, and production of charginos in photon fusion is described by the Breit-Wheeler cross section [20],

$$
\sigma\left(\gamma \gamma \rightarrow \tilde{\chi}_{1}^{+} \tilde{\chi}_{1}^{-}\right)=\frac{4 \pi \alpha^{2}}{s}\left[\left(1+\frac{4 m_{\chi}^{2}}{s}-\frac{8 m_{\chi}^{4}}{s^{2}}\right) \ln \frac{1+\sqrt{1-4 m_{\chi}^{2} / s}}{1-\sqrt{1-4 m_{\chi}^{2} / s}}-\left(1+\frac{4 m_{\chi}^{2}}{s}\right) \sqrt{1-\frac{4 m_{\chi}^{2}}{s}}\right],
$$

where $m_{\chi}$ is the chargino mass, $\sqrt{s} \equiv \sqrt{4 \omega_{1} \omega_{2}}$ is the invariant mass of the pair of charginos, $\omega_{1}$ and $\omega_{2}$ are photons energies.

Fiducial cross section takes into account kinematic cuts. For chargino production in UPC the main cuts are:

- on relative momentum loss of the colliding particles, $\xi_{\min }<\xi<\xi_{\max }$ (i.e. on the energy of the colliding photons, $\omega=\xi \cdot E$, where $E$ is the energy of the colliding particle, proton or heavy ion);

- on chargino transverse momentum, $p_{T}>\hat{p}_{T}$;

- on chargino pseudorapidity, $|\eta|<\hat{\eta}$.

\footnotetext{
${ }^{2}$ To get $n(\omega)$ one has to integrate (1) over transverse momentum $\vec{q}_{\perp}$.
} 
For the fiducial cross section we get:

$$
\begin{aligned}
\sigma_{\text {fid. }}(p p & \left.\rightarrow p p \tilde{\chi}_{1}^{+} \tilde{\chi}_{1}^{-}\right)= \\
& =\int_{\left(4 \xi_{\min } E\right)^{2}}^{\left(4 \xi_{\max } E\right)^{2}} \mathrm{~d} s \int_{\max \left(\hat{p}_{T}, \frac{\sqrt{s / 4-m_{\chi}^{2}}}{\cosh \hat{\eta}}\right)}^{\sqrt{s / 4-m_{\chi}^{2}}} \mathrm{~d} p_{T} \frac{\mathrm{d} \sigma\left(\gamma \gamma \rightarrow \tilde{\chi}_{1}^{+} \tilde{\chi}_{1}^{-}\right)}{\mathrm{d} p_{T}} \int_{1 / \hat{x}}^{\hat{x}} \frac{\mathrm{d} x}{8 x} n\left(\sqrt{\frac{s x}{4}}\right) n\left(\sqrt{\frac{s}{4 x}}\right),
\end{aligned}
$$

where $x=\omega_{1} / \omega_{2}$, and

$$
\hat{x}=\left(\hat{X}+\sqrt{\hat{X}^{2}+1}\right)^{2}, \hat{X}=\frac{\sqrt{s} p_{T}}{2\left(p_{T}^{2}+m_{\chi}^{2}\right)}\left(\sinh \hat{\eta}-\sqrt{\cosh ^{2} \hat{\eta}+\frac{m_{\chi}^{2}}{p_{T}^{2}}} \cdot \sqrt{1-\frac{4\left(p_{T}^{2}+m_{\chi}^{2}\right)}{s}}\right) .
$$

The differential with respect to $p_{T}$ cross section is

$$
\frac{\mathrm{d} \sigma\left(\gamma \gamma \rightarrow \tilde{\chi}_{1}^{+} \tilde{\chi}_{1}^{-}\right)}{\mathrm{d} p_{T}}=\frac{8 \pi \alpha^{2} p_{T}}{s\left(p_{T}^{2}+m_{\chi}^{2}\right)} \cdot \frac{1-\frac{2\left(p_{T}^{4}+m_{\chi}^{4}\right)}{s\left(p_{T}^{2}+m_{\chi}^{2}\right)}}{\sqrt{1-\frac{4\left(p_{T}^{2}+m_{\chi}^{2}\right)}{s}}} .
$$

Let us calculate corresponding cross sections for the LHC parameters. For $m_{\chi}=100 \mathrm{GeV}$, pp collision energy $13 \mathrm{TeV}, \mathrm{PbPb}$ collision energy 5.02 TeV/(nucleon pair), we get

- $\sigma\left(p p \rightarrow p p \tilde{\chi}_{1}^{+} \tilde{\chi}_{1}^{-}\right)=2.84 \mathrm{fb}$,

- $\sigma\left(\mathrm{Pb} \mathrm{Pb} \rightarrow \mathrm{Pb} \mathrm{Pb} \tilde{\chi}_{1}^{+} \tilde{\chi}_{1}^{-}\right)=21.2 \mathrm{pb}$. Therefore, for $2.4 \mathrm{nb}^{-1}$ of integrated luminosity there are only 0.053 events.

For the fiducial cross section with parameters

$$
\begin{array}{rlrl}
m_{\chi} & =100 \mathrm{GeV}, & E & =6.5 \mathrm{TeV}, \\
\xi_{\text {min }} & =0.015, & \xi_{\max } & =0.15, \\
\hat{p}_{T} & =20 \mathrm{GeV}, \quad \hat{\eta} & =2.5,
\end{array}
$$

we get

$$
\sigma_{\text {fid }}\left(p p \rightarrow p p \tilde{\chi}_{1}^{+} \tilde{\chi}_{1}^{-}\right)=0.72 \mathrm{fb} .
$$

For a heavy ion to hit the forward detector, its energy loss has to be at least $7.8 \mathrm{TeV}$. Therefore, fiducial cross section is suppressed by both the Breit-Wheeler cross section and nucleus form factor. But it is still possible to look for chargino in UPC with the help of Eloss and TOF methods if there will be enough statistics.

In what follows we consider only $p p$ events since at the present moment the expected number of events in heavy ions is to small.

\section{Mass reconstruction}

In case of UPC the event kinematics can be fully reconstructed by measuring the proton energies in forward detectors. In what follows we will study the advantages provided by this feature of UPC. The method proposed can be complemented by the conventional $\mathrm{d} E / \mathrm{d} x$ and TOF measurements.

Momenta of all particles in the final state can be measured: 
- momenta of chargino candidates $\vec{p}_{1}, \vec{p}_{2}$ can be reconstructed from the tracks in the detector;

- final state protons can be detected by the forward detectors measuring $\xi_{1}, \xi_{2}$.

These four values are sufficient to reconstruct the chargino candidates mass ${ }^{3}$ :

$$
m=\sqrt{\frac{\left(2 \xi_{1} \xi_{2} E^{2}+\vec{p}_{1} \vec{p}_{2}\right)^{2}-\vec{p}_{1}^{2} \vec{p}_{2}^{2}}{4 \xi_{1} \xi_{2} E^{2}+\left(\vec{p}_{1}+\vec{p}_{2}\right)^{2}}}
$$

Calculating this value for the events with two charged tracks and two protons in forward detectors one should obtain the delta-function at the chargino mass smeared with the detector resolution.

\section{Background}

Since we suggest to detect both protons and charginos, and require that the latter hit the muon system, there are two possible kinds of background sources:

- Production of $\mu^{+} \mu^{-}$pairs. The main sources of muons in UPC are the following reactions:

$$
\begin{aligned}
& \text { 1.pp } \rightarrow p p \mu^{+} \mu^{-} . \\
& \text {2.pp } \rightarrow p p W^{+} W^{-} \rightarrow p p \mu^{+} v_{\mu} \mu^{-} \bar{v}_{\mu} . \\
& \text { 3.pp } \rightarrow p p \tau^{+} \tau^{-} \rightarrow p p \mu^{+} v_{\mu} \bar{v}_{\tau} \mu^{-} \bar{v}_{\mu} v_{\tau} .
\end{aligned}
$$

The result of Monte Carlo simulation of the signal and background processes for parameters (7) is shown in Fig. 1a. In the simulation the finite detector resolution is taken into account in accordance with [21, Section 4.5], see [16] for details. It is worth noting that $W W$ and $\tau \tau$ backgrounds can be further suppressed by imposing the cut on the transverse momentum conservation, however there is no need in that since these backgrounds are small.

- Pile-up events producing protons in the forward detectors. The main process here is proton diffractive dissociation. These protons in combination with large background from soft $\mu \mu$ production in UPC (since muons are soft, protons from UPC are not detected) significantly increase the background. The spectrum of protons from proton diffractive dissociation is well known [22-24]. The result of Monte Carlo simulation with the account of this background (see [16] for details) is shown in Fig. 1 b.

This large background can be suppressed by imposing an additional cut on longitudinal momenta:

$$
\left|p_{\|, 1}+p_{\|, 2}-\left(\xi_{1}-\xi_{2}\right) E\right|<\hat{p}_{\|} .
$$

The effect of this cut with $\hat{p}_{\|}=20 \mathrm{GeV}$ is shown in Fig. 1c.

The results for $m_{\chi}=150 \mathrm{GeV}$ with the account of all background sources and the cut (10) are shown in Fig. 2. This is a good example, since it gives the idea of what masses are accessible with the method described: the significance of this peak ${ }^{4}$ is just a little less than 5 and the significance will be 3 if the trigger and reconstruction efficiency will be at the level of 0.4 as it was estimated in [25].

\footnotetext{
${ }^{3}$ It is worth noting that different formulas can be derived for this value, see [16]. They are equivalent up to the momentum conservation law. However, since all parameters are measured with finite accuracy, these formulas can perform differently for the same experimental data. Here we have chosen one that performs better.

${ }^{4}$ See how it was estimated in [16].
} 


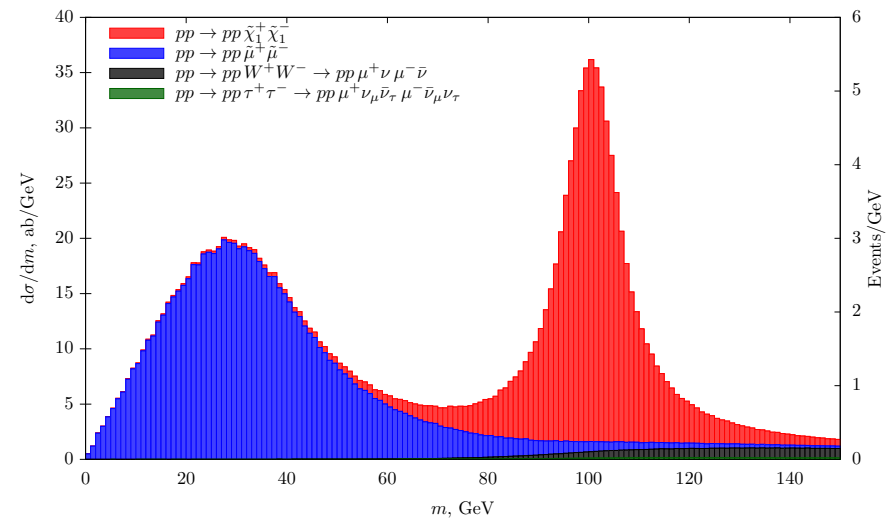

(a) Signal and background events with the account of finite detector resolution.

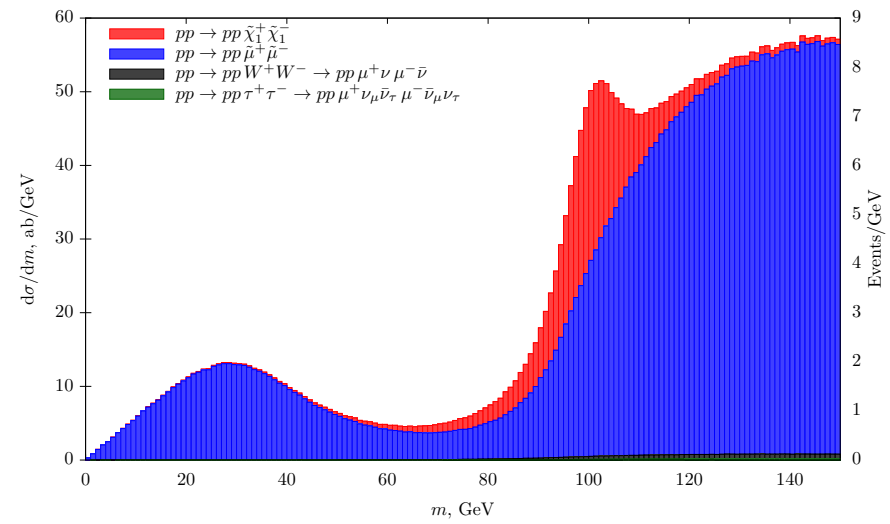

(b) The proton diffractive dissociation is taken into account.

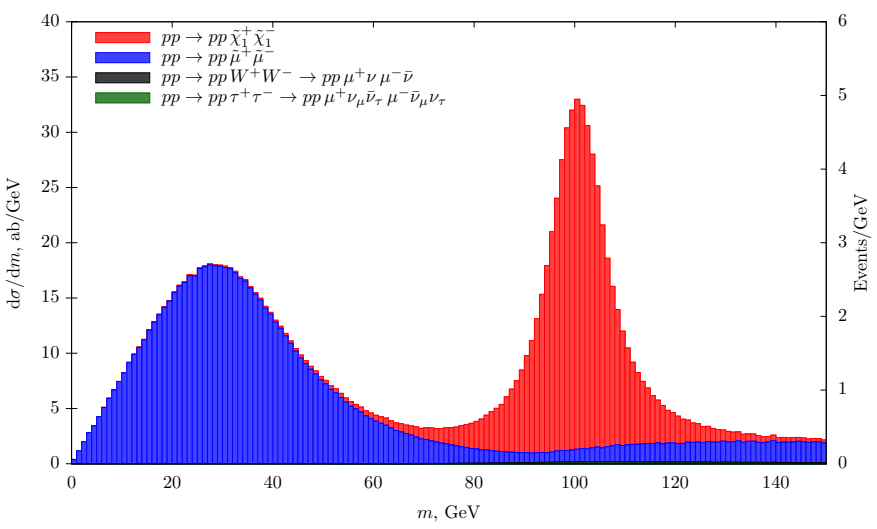

(c) The effect of additional cut on parallel momenta (10).

Figure 1: Results of Monte Carlo simulation for parameters (7). The integrated luminosity is assumed to be $150 \mathrm{fb}^{-1}$. The bin width is $1 \mathrm{GeV}$. 


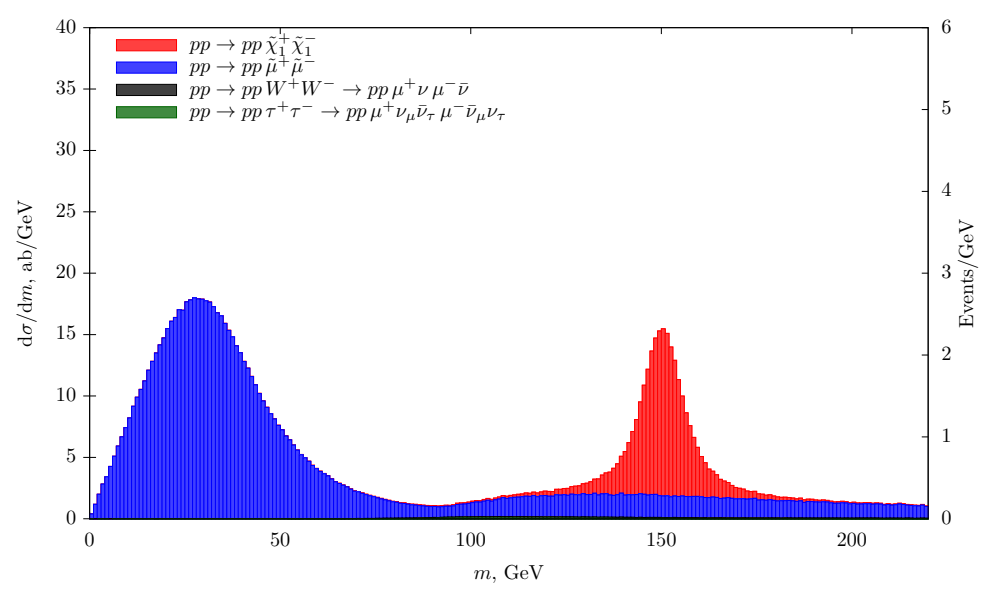

Figure 2: The same as Fig. 1c but for $m_{\chi}=150 \mathrm{GeV}$.

\section{Conclusions}

Ultraperipheral collisions provide us with a model-independent method for New Physics searches in photon-photon fusion. Detection of both protons in forward detectors allows for full kinematics reconstruction providing a way to greatly suppress the background.

The case of quasistable chargino was considered and it was shown that charginos with the mass up to $150 \mathrm{GeV}$ can be found in $p p$ collisions with already available LHC data.

To find charginos in heavy ion collisions radical increase of luminosity is required.

\section{Acknowledgments}

The authors are supported by the Russian Science Foundation grant No 19-12-00123.

\section{References}

[1] M. Tanabashi et al. (Particle Data Group), Review of Particle Physics, Phys. Rev. D98, 030001 (2018)

[2] The CMS Collaboration, JHEP 1103, 024 (2011), arXiv:1101.1645

[3] The ATLAS Collaboration, Phys. Lett. B703, 428 (2011), arXiv:1106.4495

[4] The CMS Collaboration, Phys. Lett. B713, 408 (2012), arXiv:1205.0272

[5] The CMS Collaboration, JHEP 1307, 122 (2013), arXiv:1305.0491

[6] The ATLAS Collaboration, JHEP 1501, 068 (2015), arXiv:1411.6795

[7] The CMS Collaboration, Eur. Phys. J. C75, 325 (2015), arXiv:1502.02522

[8] The ATLAS Collaboration, Eur. Phys. J. C75, 407 (2015), arXiv:1506.05332

[9] The LHCb Collaboration, Eur. Phys. J. C75, 595 (2015), arXiv:1506.09173

[10] The ATLAS Collaboration, Phys. Rev. D93, 112015 (2016), arXiv:1604.04520

[11] The CMS Collaboration, Phys. Rev. D94, 112004 (2016), arXiv:1609.08382

[12] The ATLAS Collaboration, Phys. Lett. B788, 96 (2019), arXiv:1808.04095

[13] The ATLAS Collaboration, Phys. Rev. D99, 092007 (2019), arXiv:1902.01636

[14] The ATLAS Collaboration, ATLAS Forward Proton Phase I Upgrade. Technical Design Report, CERN-LHCC-2015-009, ATLAS-TDR-024-2015 
[15] The CMS and TOTEM Collaborations, CMS-TOTEM Precision Proton Spectrometer. Technical Design Report, CERN-LHCC-2014-021, TOTEM-TDR-003

[16] S. I. Godunov, V. A. Novikov, A. N. Rozanov, M.I. Vysotsky, E. V. Zhemchugov, arXiv: 1906.08568

[17] M. Vysotsky, E. Zhemchugov, Usp.Fiz.Nauk 189, 975-984 (2019), arXiv:1806.07238

[18] S. Pacetti, R. B. Ferroli, E. Tomasi-Gustafsson, Phys.Rep. 550, 1 (2015)

[19] B. Dreher, J. Friedrech, K. Merle, H. Rothhaas, G. Lührs, Nucl.Phys. A235, 219 (1974)

[20] G. Breit, J. A. Wheeler, Phys.Rev. 46, 1087 (1934)

[21] ATLAS Inner Detector Community, Technical Design Report vol. I, ATLAS-TDR-4, CERN/LHCC 97-16 (1997)

[22] A. B. Kaidalov, Phys. Rep. 50, 157 (1979)

[23] A. B. Kaidalov, V. A. Khoze, Yu. F. Pirogov, N. L. Ter-Isaakyan, Phys. Lett. B45, 493 (1973)

[24] L. A. Harland-Lang, V. A. Khoze, M. G. Ryskin, M. Tasevsky, JHEP 1904, 010 (2019), arXiv: 1812.04886

[25] The ATLAS Collaboration, Phys.Lett. B 777, 303 (2018), arXiv:1708.04053 